\title{
Reducción del Tiempo de Ciclo de Inyección de Termoplásticos con el uso de Moldes con Tratamiento Superficial por Nitruración
}

\author{
Emerson J. Corazza ${ }^{(1)}$, Carlos M. Sacchelli ${ }^{(2)}$ y Cintia Marangoni ${ }^{(1)_{*}}$ \\ (1) Universidade da Região de Joinville - UNIVILLE, Mestrado em Engenharia de Processos, \\ Rua Paulo Malschitzki 10, Distrito Industrial, CEP: 89219-710, Joinville, SC-Brasil \\ (e-mail: ej.corazza@gmail.com; cintia.marangoni@univille.br) \\ (2) Universidade Federal de Santa Catarina - UFSC, Centro de Engenharia da Mobilidade, \\ Rua Paulo Malschitzki 10, Distrito Industrial, CEP: 89219-710, Joinville, SC-Brasil \\ (e-mail: carlos.sacchelli@gmail.com) \\ * Autor a quien debe ser dirigida la correspondencia
}

Recibido Jul. 21, 2011; Aceptado Sep. 30, 2011; Versión final recibida Nov. 23, 2011

\section{Resumen}

Se presenta un estudio sobre la transferencia de calor en un molde, comparando los ensayos de inyección utilizando moldes con y sin tratamiento superficial de nitruración. Simulaciones del proceso se realizaron con el uso de Asistencia Computacional a Ingeniería (CAE) para determinar los parámetros iniciales que se aplicaron en los ensayos reales en moldes instrumentados de acero P20 (con y sin tratamientos), inyectados con el polímero poliestireno cristal. Los resultados indicaron una reducción del tiempo de enfriamiento y del tiempo de proceso en el molde con el tratamiento, debido a una mejora en la conductividad térmica.

\section{Cycle Time Reduction of Thermoplastic Injection using Nitriding Treatment Surface Molds}

\begin{abstract}
A study on the heat transfer in a mold, comparing experimental injection tests using a mold with and without surface treatment (nitriding) is presented. Process simulations were carried out using Computer Aided Engineering tools (CAE) for the estimation of the initial parameters to be applied in the tests in instrumented P20 steel injection molds with the polymer crystal polystyrene. Results indicated a reduction of cooling time and of the time of the process in the mold with treatment, due to an increase of the thermal conductivity.
\end{abstract}

Keywords: injection, mold, cycle time, cooling, surface treatment 


\section{INTRODUCCIÓN}

El fuerte incremento en el uso de productos fabricados con materiales termoplásticos que van desde artículos para el hogar a sofisticadas piezas aeronáuticas, aumentó la competencia dentro de este mercado de manera significativa. Las aplicaciones parecen ilimitadas para los termoplásticos, pudiendo hacerse desde piezas simples a muy complejas con una grande tolerancia dimensional (Ozdemir et al., 2004). Numerosos artículos de uso cotidiano tienen una pieza moldeada por el proceso de inyección, entonces las empresas vinculadas a este proceso sufren exigencias para constante innovación de los productos mejorando sus técnicas y aumentando la calidad (Bareta et al., 2008; Fuh et al., 2004).

El ciclo del proceso de inyección tiene cuatro etapas bien caracterizadas: (1) el calentamiento del termoplástico, (2) la entrada de material polimérico en el molde, (3) la transferencia de calor o refrigeración y (4) el desmoldeo de la pieza. Las propiedades e calidad de los moldes determinan la productividad del proceso, ya que la tasa de producción, es decir el tiempo del ciclo, dependerá de la rapidez con que el material puede ser calentado, inyectado, solidificado y expulsado. Entre estas etapas, el enfriamiento es la que demanda mayor tiempo, así es fundamental un bueno proyecto del sistema no sólo para reducir el tiempo de ciclo de proceso, sino también para mejorar la calidad del producto (Hassan et al., 2009), que puede ser clasificada como calidad externa: dimensión, forma, propiedades mecánicas, eléctricas y ópticas y calidad interna: tensión de cizallamiento, peso y orientación molecular y grado de cristalización (Wong et al., 2008). Un incorrecto enfriamiento puede llevar a deformaciones conocidas como rechupes y indeseables tensiones residuales (Hassan et al., 2010).

El proyecto del sistema de refrigeración debe ser capaz de eliminar el calor a una tasa deseada de forma eficiente y suficiente para que la pieza plástica pueda ser eyectada sin distorsión (Li et al., 2004). Un sistema eficaz reduce al mínimo los efectos adversos y promueve la distribución de la temperatura uniforme a través de la pieza, lo que resulta en un tiempo mínimo de enfriamiento (Bom y Leães, 2008; Dimla et al., 2005).

El aumento de la calidad y la productividad combinada con una mayor demanda en el área de inyección de termoplásticos hace que los proyectos de desarrollo de moldes deban ser cada vez más efectivos y responsables de la ejecución adecuada y efectiva del molde, sus componentes y el producto final. En este sentido, el objetivo de una reducción en el tiempo del ciclo de molde por inyección de termoplásticos, son temas de estudios como el de Postawa et al., (2008) que reducen los tiempos del ciclo de inyección mejorando la transferencia del calor. El proyecto del molde con los materiales y dimensiones apropiadas para el sistema de refrigeración hará que el ciclo de enfriamiento se reduzca, como así también el costo final del componente (Cátic et al., 2006).

Junto con el tema del proyecto óptimo de los canales de refrigeración del molde, los tratamientos de superficie se emplean con el fin de mejorar tanto las propiedades (durabilidad, resistencia a la corrosión, al desgaste y a la fatiga térmica) y la optimización del flujo de calor, ya que propiedades tales como la conductividad térmica del material se cambian (Moreno et al., 2005). Entre los diferentes tipos de tratamientos de superficie, el más ampliamente utilizado es la nitruración debido a la simplicidad, costo relativamente bajo y la eficiencia. Sin embargo, su aplicación es todavía poco explorada en relación con las ganancias que puede ofrecer el ciclo del proceso, especialmente en relación con el sistema de refrigeración (Ferreira, 2001).

Para el auxilio en la mejora del proyecto de moldes son utilizados herramientas CAE, que debido a lo nivel de confianza adquirido por ellas ha permitido utilizarlas no sólo para optimizar el flujo en procesos de inyección de plásticos, sino que incluso se han empleado para validar resultados de otras investigaciones (López et al., 2006). En este trabajo fui utilizada esta herramienta para obtenciones de valores iniciales del proceso de inyección.

Por lo tanto, este trabajo pretende realizar un estudio experimental preliminar acerca de la transferencia de calor a partir de un molde. Se exploró el tiempo de enfriamiento en un ciclo de 
inyección de termoplásticos y esto se evaluó comparando un molde sin tratamiento y con otro con un tratamiento superficial de nitruración. Se comenzó realizando simulaciones para estimar los parámetros iniciales del proceso con la herramienta CAE Moldflow. Luego se llevaron a cabo ensayos de inyección con medición por sensores de temperaturas en diferentes puntos del molde para el análisis del efecto de la nitruración en el ciclo de enfriamiento del proceso.

\section{MATERIALES Y MÉTODOS}

Para este trabajo se realizaron dos insertos de acero P20 en un molde ya existente: uno no tratado (Figura 1) y otro con un tratamiento superficial de nitruración de gas. El material plástico utilizado fue el termoplástico Polystyrol 158K de BASF.

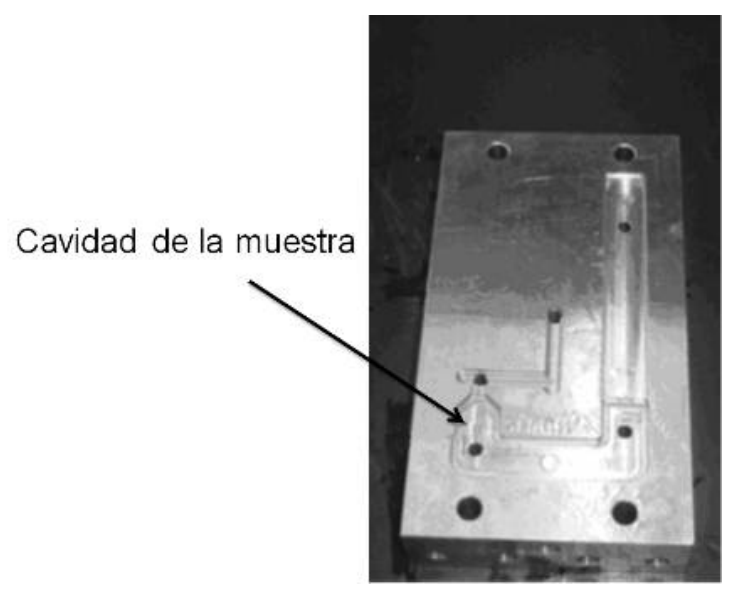

Fig. 1: Inserto con la cavidad tratada.

El tratamiento de la superficie de nitruración gaseosa se llevó a cabo en una atmósfera de $\mathrm{NH}_{3}+$ $\mathrm{CO}_{2}+\mathrm{N}_{2}$, a una temperatura de $560^{\circ} \mathrm{C}$ durante 10 horas, resultando en una profundidad de 0,30 $\mathrm{mm}$ de la capa de difusión, 0,02 mm en la capa blanca, con un aumento de la dureza de $345 \mathrm{HV}$ para $817 \mathrm{HV}$ en la superficie. La figura 2 muestra la presencia de la capa nitrurada en la superficie de la pieza. También fue realizado el ensayo de conductividad térmica pelo método flash (Norma ASTM E1461), que resulto en valore de $26,33 \mathrm{~W} / \mathrm{mK}$ para el P20 no tratado y de $28,46 \mathrm{~W} / \mathrm{mK}$ para el material tratado.

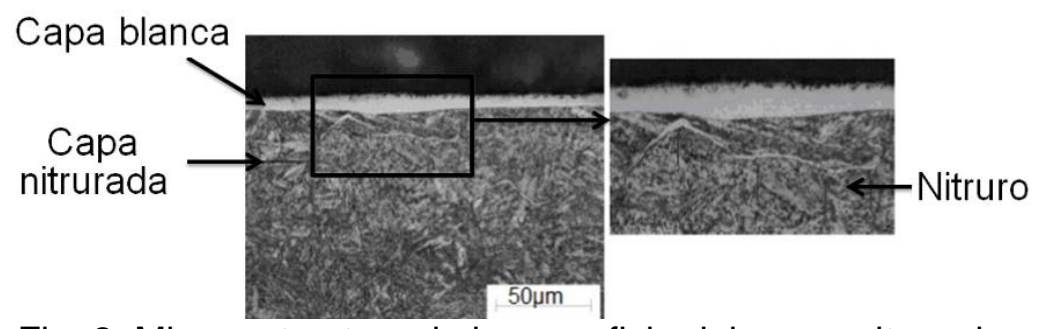

Fig. 2: Micro estructura de la superficie del acero nitrurado.

Otros equipos utilizados fueran una máquina de molde por inyección, Battenfeld 250 Plus, un molde instrumentado con seis termopares tipo $\mathrm{J}$ y un dispositivo de adquisición de datos, Yokogawa modelo DXA120. Para el proceso de refrigeración se utilizo un sistema cerrado a través de un dispositivo para circulación de agua a una velocidad de $4 \mathrm{l} / \mathrm{min}$ con control de su temperatura, que se fijó en $35^{\circ} \mathrm{C}$.

En ensayo de inyección, se evaluó las temperaturas del molde y su medidas fueron analizadas. De los seis termopares utilizados, dos estaban en el extremo de la cavidad (1 y 6 en la Figura 3), con una capa protectora de acero inoxidable estando así en contacto con el material plástico en el momento de la entrada do material en la cavidad y los otros cuatro termopares $(2,3,4$ y 5$)$ en una posición de $5 \mathrm{~mm}$ abajo de la superficie del molde en contacto con el material plástico localizados según la Figura 3. 
Durante los ensayos de inyección, las piezas se han extraído mediante el análisis de la variación de la masa. Las análisis se realizaron para la verificación y control de la presión de inyección, garantizando que la misma extraiga piezas regulares, sin desperdicios de materiales y sin fallas. Las propiedades de los materiales seleccionados para este trabajo, el tipo de inyector y las condiciones del proceso propuestos por el fabricante fueron utilizados como datos de entrada en el CAE Moldflow a través de la simulación. El método matemático de análisis Cool + Flow fue el utilizado para lo ensayo debido ser o más adecuado de acordó con Sacchelli y Cardoso (2007). Por lo tanto, inicialmente fue construido un modelo que representa la pieza plástica, como se ilustra en la Figura 4 (a) y luego los otros componentes del molde, como se muestra en la Figura 4 (b). En el modelo geométrico se realizó un mallado, empleando aproximadamente 8418 elementos triangulares con una resolución aproximada de $2 \mathrm{~mm}$.

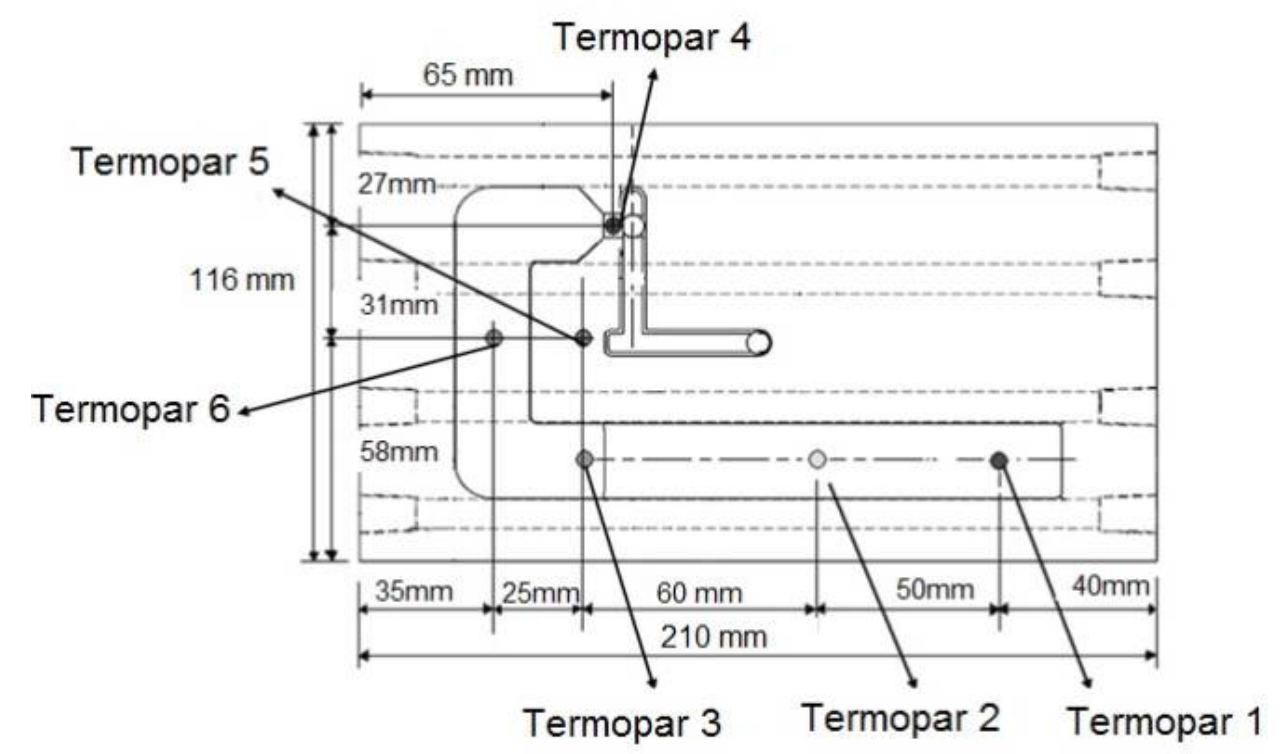

Fig. 3: Disposición de los termopares.

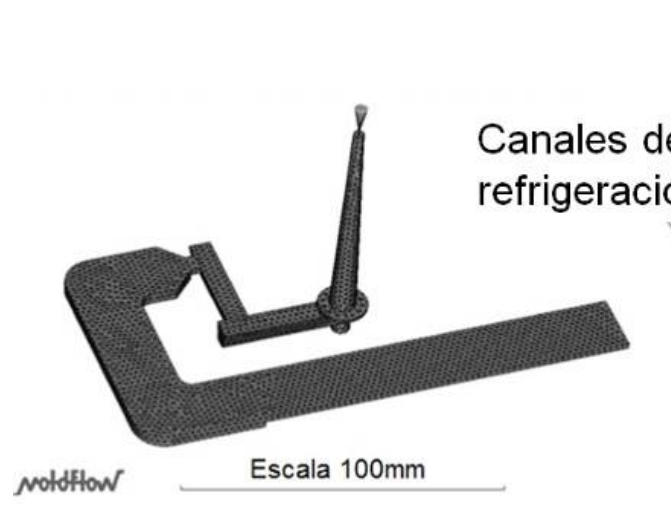

(a)

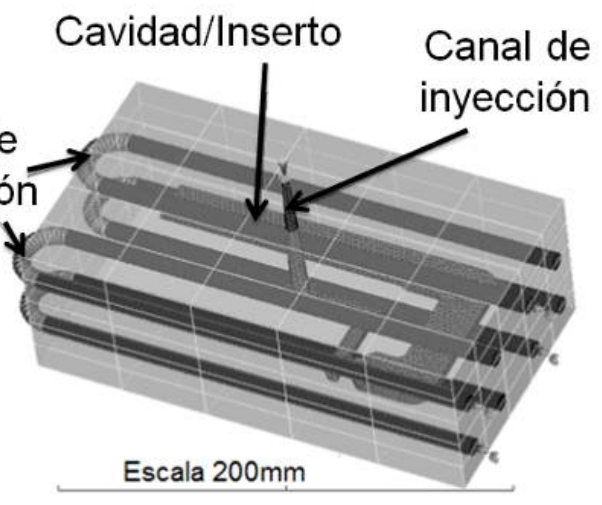

(b)

Fig. 4: (a) Modelo de la geometría de la muestra y sus canales de refrigeración, (b) Modelo de molde de inyección para simulación.

Las condiciones iniciales del proceso se generaron en las simulaciones y se presentan en la Tabla 1. En los ensayos con el molde nitrurado se utilizaron los mismos parámetros de los experimentos con el molde sin tratamiento. Pero con el molde nitrurado fue posible reducir el tiempo de enfriamiento de $30 \mathrm{~s}$ para $25 \mathrm{~s}$, tiendo el tiempo de ciclo de la máquina reducido de $42 \mathrm{~s}$ para $37 \mathrm{~s}$. 
Tabla 1: Parámetros utilizados en la simulación de la inyección.

\begin{tabular}{|l|c|}
\hline Parámetro & Valor \\
\hline Tiempo de inyección $(\mathrm{s})$ & 3,2 \\
\hline Temperatura de inyección $\left({ }^{\circ} \mathrm{C}\right)$ & 230 \\
\hline Temperatura del molde $\left({ }^{\circ} \mathrm{C}\right)$ & 40 \\
\hline Temperatura del refrigerante $\left({ }^{\circ} \mathrm{C}\right)$ & 43,5 \\
\hline Tiempo de apertura del molde $(\mathrm{s})$ & 5 \\
\hline Tiempo de ciclo de la máquina $(\mathrm{s})$ & 42 \\
\hline Temperatura ambiente $\left({ }^{\circ} \mathrm{C}\right)$ & 26 \\
\hline Fuerza de cierre $($ Kgf $)$ & $7.10^{3}$ \\
\hline Flujo de agua de refrigeración $(\mathrm{l} / \mathrm{min})$ & 4,17 \\
\hline Tiempo de enfriamiento $(\mathrm{s})$ & 30 \\
\hline
\end{tabular}

\section{RESULTADOS Y DISCUSIONES}

En los ensayos de inyección que duraron cerca de 1 h 15 min utilizando dos tipos de molde, un molde sin tratamiento y un molde con tratamiento fueron tomadas las temperaturas en seis puntos, como se indica en la Figura 2.

El tiempo de enfriamiento regulado en la máquina de inyección es el tiempo requerido para la pieza que se extrae sin mostrar una deformación significativa. Para el molde con el tratamiento fue posible reducir en 5 segundos el tiempo, esto representa un gano de cerca de 17\% no tiempo de enfriamiento e $12 \%$ no ciclo total de inyección. Con esta reducción se puede fabricar cerca de 12 piezas a mas en una hora.

La temperatura de lo fluido refrigerante (agua) en el molde sin tratamiento vario de $43{ }^{\circ} \mathrm{C}$ a $44{ }^{\circ} \mathrm{C}$. En el molde nitrurado lo fluido vario de $39^{\circ} \mathrm{C}$ a $40^{\circ} \mathrm{C}$. Fue observado, que con la misma fuente de calor (temperatura de inyección de $230^{\circ} \mathrm{C}$ ), una variación en la transferencia de calor cuando se comparan el molde tratado con el no tratado. Los perfiles de la temperatura del fluido se presentan en la Figura 5.

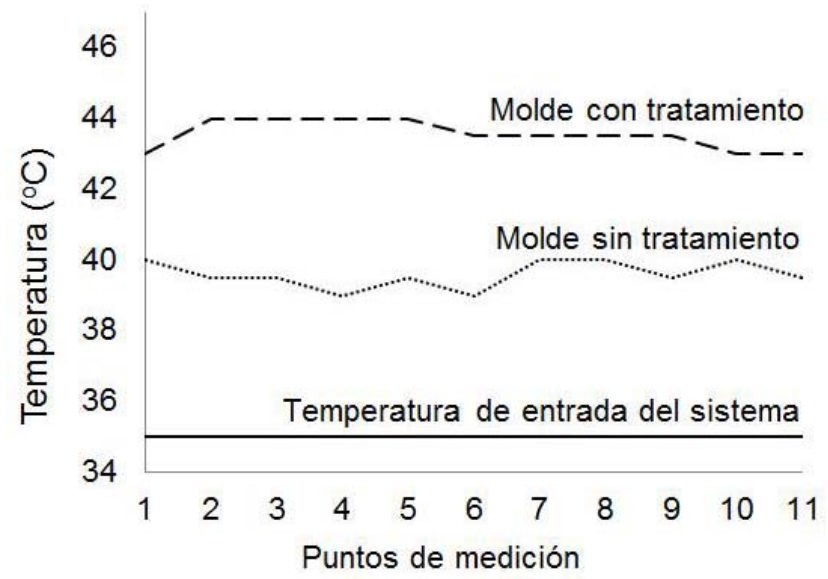

Fig. 5: Perfil de temperatura del agua de refrigeración durante los ensayos.

El tiempo de toma de datos de temperatura durante el ciclo de inyección fue a cada 2 segundos. El primer ensayo generó un total de 2215 valores para cada termopar. Durante los ensayos experimentales se ha observado que la temperatura del refrigerante varió desde 39 hasta $44{ }^{\circ} \mathrm{C}$. Por lo tanto, se decidió realizar el análisis en el momento en que la temperatura del fluido se mantuvo estable durante más tiempo. La Figura 6 (a) muestra los perfiles de las variaciones de temperatura en el período seleccionado de tiempo. 
Las mismas mediciones se realizaron en lo segundo ensayo de la inyección, resultando en 2319 los valores para cada termopar. También fue analizado el periodo en que lo fluido refrigerante se mantuvo mas estable. La Figura 6 (b) muestra la variación.

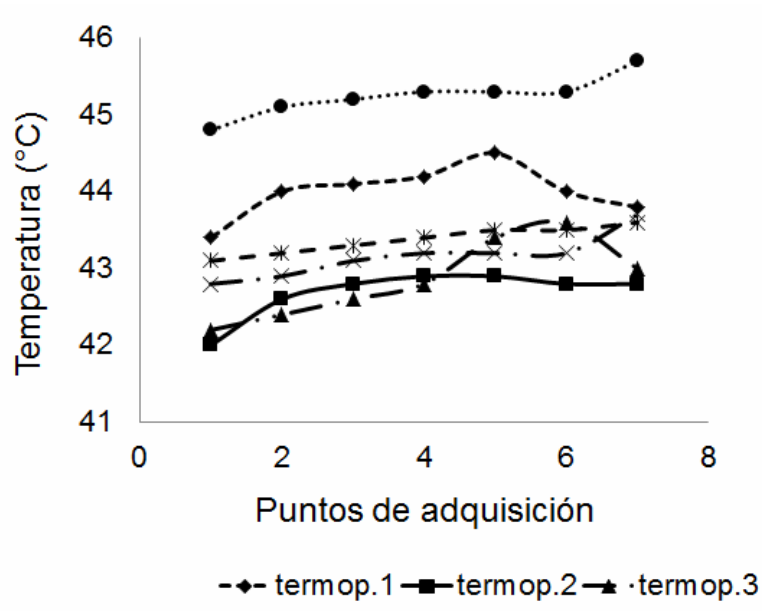

(a)

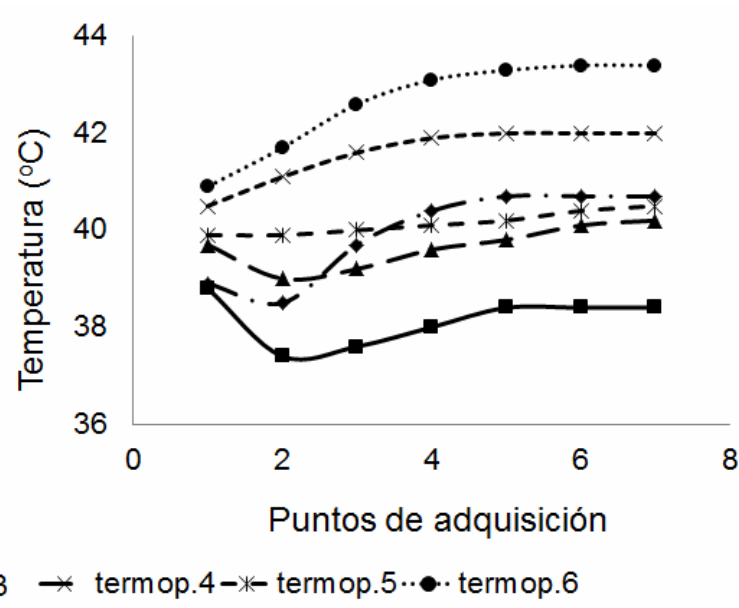

(b)

Fig. 6: Perfiles de los cambios de temperatura de los ensayos usando el molde (a) sin tratar y (b) con el tratamiento.

Para una analize entre os dados de temperatura se considero el valor máximo de temperatura obtido en los intervalos da figura 6 (a) e (b). En la Figura 7 se muestran las diferencias entre los puntos, en comparación con los ensayos en el molde sin tratamiento y tratados en la superficie del molde.

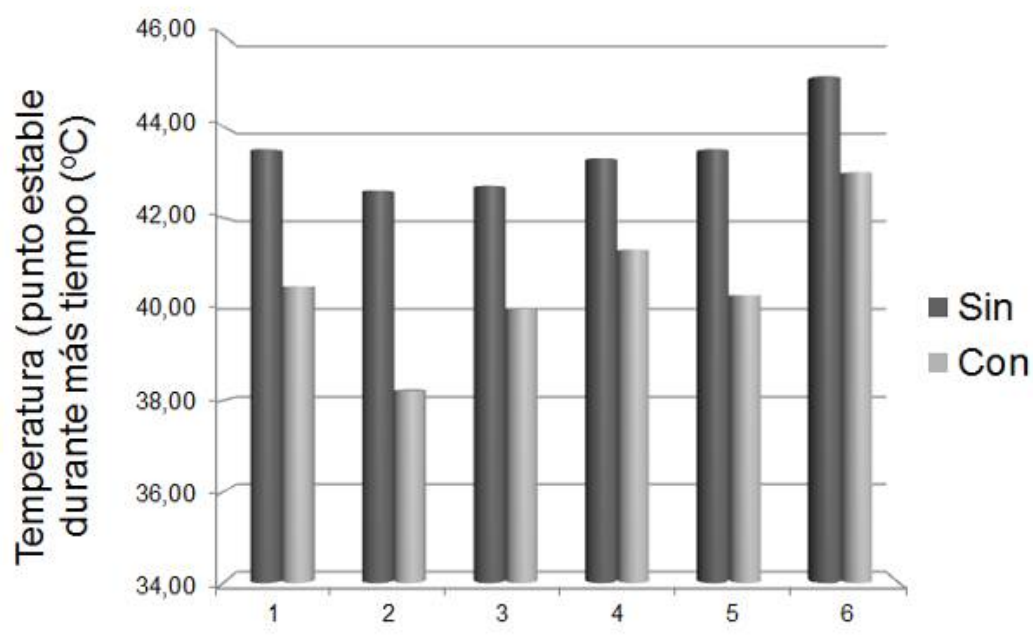

Puntos de adquisición de temperaturas

Fig. 7: Gráfico comparativo de las temperaturas en los termopares en el momento en que la temperatura del fluido se mantuvo estable durante más tiempo.

La comparación entre los dos perfiles de temperatura presentados muestra una disminución en los valores con el molde tratado, en relación a la primera, con el molde sin tratamiento. Se observa una temperatura en la media $3,2^{\circ} \mathrm{C}$ mas baja. Esta reducción indica que como la nitruración gaseosa aumento la conductividad térmica la transferencia de calor entre o acero P20 y lo fluido aumento (se puede ver debido al aumento de la temperatura del fluido) reduciendo así el tiempo de enfriamiento, que reduce el tiempo total del ciclo de inyección.

Los datos obtenidos se compararon posteriormente con las simulaciones de Moldflow para validar el uso de este aplicativo, tanto en el molde sin tratamiento como en el molde nitrurado. 


\section{CONCLUSIONES}

En este estudio se encontró que un molde nitrurado tiene un más eficiente transferencia de calor que un molde sin tratar. Esta diferencia hace que un molde nitrurado tenga una productividad mayor que un molde sin tratamiento. En nuestro estudio el molde sin tratamiento requería 42 segundos para la producción de cada pieza mientras que el molde nitrurado solo requería 37 segundos por pieza. Nuestra conclusión es que la superficie nitrurada aumento la conductividad térmica y con esto una meloja de la transferencia de calor entre o acero P20 y o fluido refrigerante. En consecuencia el ciclo de enfriamiento se torna más eficiente resultando en una productividad mayor.

\section{REFERENCIAS}

Bareta, D. R., C. A. Costa, A. J. Zattera y A. S. Pouzada, Influência de materiais alternativos nas propriedades de peças tubulares no contexto de moldes protótipos de injeção, Tecnologia em Metalurgia e Materiais: 4(3),37-42 (2008).

Bom, P. R. y V. S. Leães, Avaliação do empenamento de peças plásticas injetadas em um molde com diferenças de temperatura entre as placas, Estudos Tecnológicos: 4(2), 135-145 (2008).

Cátic, I., M. R. Sokele y M. Sercer, Qual é a relação entre o material da cavidade e o tempo de resfriamento de peças injetadas, Plástico Industrial: 8(91), 34-41 (2006).

Dilmla, D. E., M. Camilotto y F. Miani, Design and optimization of conformal cooling channels in injection moulding tools, Journal of Materials Processing Technology: 164-165, 1294-1300 (2005).

Ferreira, G. E. F.; Avaliação da resistência a fadiga térmica do aço AISI H13 nitretado pelo processo gasoso e por plasma, Tese de Magister, Depto Eng. de Engenharia de Materiais, UFSC, Florianópolis, Brasil (2001).

Fuh, J. Y. H., Y. F. Zhang, A. Y. C. Nee y M. W. Fu, Computer-Aided Injection Mold Design and Manufacture, Marcel Dekker, New York, USA (2004).

Hassan, H., N. Regnier, C. Lebot, C. Pujos y G. Defaye, Effect of cooling system on the polymer temperature and solidification during injection molding, Applied Thermal Engineering: 29, 17861791 (2009).

Hassan, H., N. Regnier, C. Le Bot y G. Defaye, 3D study of cooling system effect on the heat transfer during polymer injection molding, International Journal of Thermal Sciences: 49, 161-169 (2010).

Li, C.L., C.G. Li y A. C. K. Mok, Automatic layout design of plastic injection mould cooling system, Computer-Aided Design: xx, 1-18 (2004).

López, F., J. Baselga y J. Bravo, Utilización de Herramientas CAE para la Determinación de Dimensiones Criticas de Lentes Realizadas por Inyección de Plásticos, Información Tecnológica: 17(4), 59-63 (2006).

Moreno, A., M. V. Leite y P. C. Borges, Efecto de la nitruración a plasma en el endurecimento por precipitación del acero 15-5 PH, Información Tecnológica: 16, 35-39 (2005).

Ozdemir, A., O. Uluer, y A. Guldas, Flow front advancement of molten thermoplastic materials during filling stage of mold cavity, Polymer Testing: 23, 957-966 (2004). 
Postawa, P., D. Kwiatkowski y E. Bociaga, Influence of the method of heating/ cooling moulds on the properties of injectin moulding parts, International Scientific Journal - Archives of Materials Science and Engineering: 31(2), 121-124 (2008).

Sacchelli, C.M. y C. A. Cardoso, El análisis de la simulación de la refrigeración del proceso de inyección de termoplásticos, $8^{\circ}$ Congreso Iberoamericano de Ingeniería Mecánica, Cusco, Peru, 23 a 27 de outubro (2007).

Wong, H. Y, K. T. Fung y F. Gao, Development of a transducer for in-line and through cycle monitoring of key process and quality variables in injection molding, Sensors and Actuators A: 141, 712-722 (2008). 
